\title{
3D Radar Objects Tracking and Reflectivity Profiling
}

\author{
Yong Hyun Kim, Hansoo Lee and Sungshin Kim* \\ Department of Electrical Engineering, Pusan National University, Busan, 609-735, Korea
}

\begin{abstract}
The ability to characterize feature objects from radar readings is often limited by simply looking at their still frame reflectivity, differential reflectivity and differential phase data. In many cases, time-series study of these objects' reflectivity profile is required to properly characterize features objects of interest. This paper introduces a novel technique to automatically track multiple 3D radar structures in C,Sband in real-time using Doppler radar and profile their characteristic reflectivity distribution in time series. The extraction of reflectivity profile from different radar cluster structures is done in three stages: 1. static frame (zone-linkage) clustering, 2. dynamic frame (evolutionlinkage) clustering and 3. characterization of clusters through time series profile of reflectivity distribution. The two clustering schemes proposed here are applied on composite multi-layers CAPPI (Constant Altitude Plan Position Indicator) radar data which covers altitude range of 0.25 to $10 \mathrm{~km}$ and an area spanning over hundreds of thousands $\mathrm{km}^{2}$. Discrete numerical simulations show the validity of the proposed technique and that fast and accurate profiling of time series reflectivity distribution for deformable 3D radar structures is achievable.
\end{abstract}

Keywords: 3D Radar Object Tracking, Doppler Weather Radar, Clustering Algorithm, Feature Extraction, Fuzzy Logic.

\section{Introduction}

Ground based radar systems are essential tool for real-time monitoring of rapidly developing aerial events and in assessing potential threat level posed by these events in the coming hours or days. Thus, the ability to accurately track and characterize radar objects in question for object classification is of particular importance in interpreting the aerial event for the strategic decision making that follows. The radar objects of interest that are covered within the scope of this paper range anything from amorphous weather clouds, thunder storms, airplane chaff, nonweather related hazardous fallout such as volcanic ash cloud [1] and other non-generic anomalous noises, etc. The genesis and time evolution characteristics of these deformable radar structures have an implication in revealing the nature of these radar objects and assessing threats based on situational awareness. In case of storm cells, the primary goal of 4D visualization and interpretation process is to locate and track already formed or potentially emerging storm cells in sequential radar images, and identify key features that provide clues to determine the storm severity and likelihood of producing microbursts. One such key feature is cross-sectional cluster height versus time profile of storm cell reflectivity. This type of profile can provide some indications of how to identify the end stage of the storm cell's life cycle, during which microburst and other potentially damaging weather events can occur. As for estimating the rainfall rate of storm cells based on both single and dual polarization radar measurements, different algorithms are used; namely, reflectivity (Zh), differential

Manuscript received Mar. 28, 2012; revised Dec. 11, 2012; accepted Dec. 14, 2012.

*corresponding author : Sungshin Kim (sskim@pusan.ac.kr) This work was supported by a 2-Year Research Grant of Pusan National University. reflectivity (Zdr), and specific differential phase $\left(K_{d p}\right)$. The typical rain algorithms are thus of the form $R\left(Z_{h}\right), R\left(Z_{h}, Z_{d r}\right)$, $R\left(K_{d p}\right)$ and $R\left(K_{d p}, Z_{d r}\right)$ depending on the type of measurement used [2].For X-band, $R\left(Z_{h}\right)$ and $R\left(Z_{h}, Z_{d r}\right)$ estimation suffer from attenuation effect of backscatter power signal while $R\left(K_{d p}\right)$ estimation does not. Therefore $Z_{h}$ and $Z_{d r}$ need to be corrected prior its usage in quantitative analysis. In this paper, corrected reflectivity values of $Z_{h}(\mathrm{CZ}$ radar volume data where $\mathrm{CZ}$ is radar volume label) which is in unit of $\mathrm{dBZ}$, is used as the basis to track radar objects and is related to rainfall rate $R\left(Z_{h}\right)(\mathrm{mm} / \mathrm{hr})$ by the following equations:

$$
\begin{aligned}
& R\left(Z_{h}\right)=\left(\frac{1}{200} 10^{\left(\frac{Z_{h}}{10}\right)}\right)^{0.625} \\
& Z_{h}=10 \cdot \log _{10}\left(200 \cdot R^{1.6}\right)
\end{aligned}
$$

The rainfall rate calculation in (1) and the reflectivity calculation in (2) -conversion from rainfall rate -are based on several equations which have been proven for more than decades of observation.

However, the difficulty of tracking and dynamically profiling cloud structures arises from the fact that these objects' morphological features are not so easily definable in both still frame and time domain. Due to their highly deformable as well as splitting and merging nature over time, the overall cloud structure set is defined in a topological space where the idea of closeness, or limits, is described in terms of relationship between sets rather than in terms of simple Euclidean distance. To mitigate this difficulty, previous tracking methods of amorphous cloud structures make the use of spatial and temporal relaxation algorithms which establish movement way points by identifying and connecting structures' localized feature points or boundary domain from 2D reflectivity 
intensity map. Spatial relaxation labeling algorithm is developed by Barnard and Thompson [3] to associate one set of feature points selected from a stereogram to another corresponding set using disparity analysis. Temporal relaxation algorithm is developed by Zhang [4] to tackle the problem of storm merging and splitting by allowing single storms to be matched to multiple storms in adjacent image frames. Krezeski [5] enabled Zhang's tracking algorithm to include the concept of pseudo-storm and property coherence which allows multiple features of a storm such as average intensity, storm size, velocity variance, storm shape and orientation, to be tracked over time in addition to storm center location. This paper takes rather different approach to the tracking problem by representing objects of interest as separate partitions in 3D radar images that are categorized into different reflectivity range, spatial and size groups, and associating selected partitions between subsequent image frames.

\section{Tracking System Overview}

The proposed tracking model is based on radar data transformation techniques as well as clustering algorithms. The model is implemented as a mean to generalize radar object tracking procedure in both fast and slow frames and also to render the procedure independent of the scale and morphological features of objects being tracked. The model also takes into consideration of logistical constraints inherently existing in the system such as the amount of available computational resources and time restriction. This is due to the fact that radar volume sweep is done at finite rate with noticeable time delay between each rotational sweep - weather events are only monitored at specific time intervals -and data collection, data transformation and sorting procedures, etc., must be completed within very short time window. The implementation of the proposed model is illustrated in Fig. 1.

In this implementation, prior being subjected to image data partitioning procedure, the original site specific radar data first undergoes coordinate system transformation from spherical to Cartesian system-radar data in UF file is stored in spherical coordinate system. This transformation is achieved by extracting interpolated 2D CAPPI layers from original $\mathrm{CZ}$ volume data for equally spaced altitudes and stacking up these 2D CAPPI layers one upon the other resulting in 3D Cartesian $\mathrm{CZ}$ volume data. After the 3D Cartesian volume data is constructed, it undergoes preliminary data sorting procedure which categorizes all individual $\mathrm{CZ}$ volume data points into two reflectivity (pixel intensity) range categories: belowthreshold and above-threshold reflectivity groups. The threshold $\mathrm{CZ}$ reflectivity is usually chosen from any values between -30 and $0 \mathrm{dBZ}$. This step is critical to speed up the overall tracking procedure by restricting the clustering procedure only to data points above minimal reflectivity level. The effect could dramatically reduce the clustering time in static frame. By filtering out background low intensity data points, this preliminary data sorting procedure produces sparse

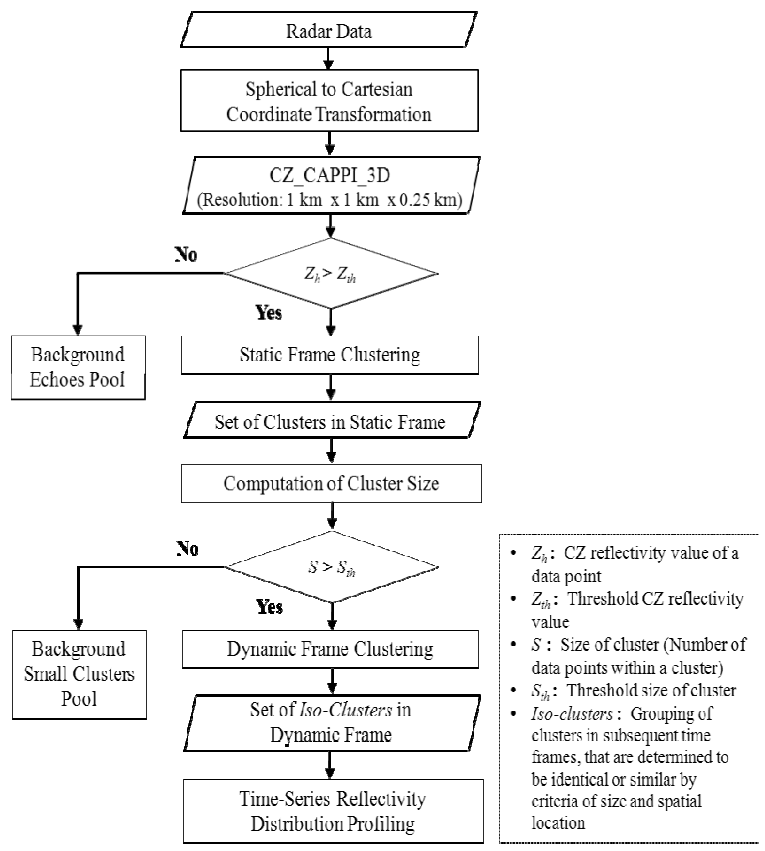

Fig. 1. Proposed model of radar objects tracking and profiling.

matrix representation of $\mathrm{CZ}$ volume data - containing only selected data points-which is then passed to static frame clustering procedure. Static frame clustering procedure, which does not require specifying the number of clusters a priori, automatically sorts out this sparse image data into multiple partitions using distance (Euclidean) similarity criteria. Resulting spatial partition s or groups from this procedure form individual cluster sets of spatially connected or neighboring data points. Once the static frame clustering procedure is completed, in order to further reduce the time taken by overall tracking algorithm, the size of each cluster-cardinality of cluster set -is computed and clusters only in select size category are chosen for dynamic clustering procedure which associates identical or similar clusters between time-series images. The output of such dynamic clustering procedure is a subspace called iso-cluster subspace where members are made up of 2tuple cluster identifiers or clusters themselves in time series that are determined to be similar by criteria of cluster size and cluster center location. This determination of the correspondence between a set of selected features objects in subsequent $3 \mathrm{D}$ radar images enables us to obtain characteristic reflectivity profiles of objects of interest in time series. The characteristic reflectivity profile of given radar object is based on frequency count versus reflectivity plot of data points within the given cluster of interest.

\section{Static Frame Clustering Method}

\subsection{Description of Zone-Linkage Clustering Method}

First comprehensive foundations on clustering techniques were published by Tryon [6] in 1939. Other introductory 
publications on clustering methods include Hartigan [7] and Murtagh [8], etc. These techniques have traditionally been applied to classification problems which are mostly concerned with problems of how to organize observed data into meaningful structures. Clustering techniques are often used in pattern recognition problems and remote sensing applications to perform image decomposition prior feature extraction procedure. The clustering procedure, in essence, partitions $N$ data values into $M$ groups so that any two data points belonging to the same group are more similar than those belonging to different groups. There are many clustering methods that exist in the current literature for image spectral analysis. However, for the purpose of fulfilling the execution time requirement of tracking procedure, a novel, time-efficient clustering method is proposed to significantly reduce the time taken by static frame clustering procedure. The novel method or zone-linkage clustering method, is loosely based on single-pass method or leader method [9] in that it falls within a nonhierarchical clustering category and all elements are treated as individual clusters at the start and at each iteration step, each element is compared with clusters formed so far and either added to the closest or used to start (lead) a new cluster if it is insufficiently close to any of the existing clusters. However, the proposed method diverges from single-pass method in its implementation and time complexity- single-pass and zone-linkage have $O\left(n^{2}\right)$ and $O(k N)$ (or simply $O(N)$ ) time complexity respectively where $k$ is the number data points enclosed by local hypersphere or neighborhood volume space to be scanned. In zonelinkage clustering, each data points in input space are processed only once for neighborhood volume scan to detect the presence of immediately neighboring data points. Detected neighborhood data points within the hyper-sphere are marked (flagged) and assigned cluster membership (added to cluster membership list). The volume scan then moves to next item in the cluster membership list until all members are processedvolume scan is performed around each of these items. Since detected data points are flagged, already marked (added) members are being skipped from being added again. If no further members are added to the cluster list, the process jumps to the next cluster and the same procedure is repeated until all data points in input space are processed. The key feature of this algorithm is that neighborhood volume scan within the hypersphere is skipped for already flagged (membership assigned) data points which dramatically improves the speed of the procedure.

\subsection{Zone-Linkage Clustering Algorithm}

Given:
\begin{tabular}{|l|l|}
\hline$\tau_{r e f}$ & Threshold reflectivity \\
$p_{i}$ & 3-tuple or coordinate point in a 3D grid (Euclidean) space \\
$r$ & Reflectivity value at $p_{i}$ \\
$S$ & Set of all coordinate points (3-tuple) in input 3D grid space \\
$A$ & Set of all coordinate points (3-tuple) with reflectivity below \\
& $\begin{array}{l}\text { threshold value } \tau_{\text {ref }} \\
B\end{array}$ \\
& $\begin{array}{l}\text { Set of all coordinate points (3-tuple) with reflectivity above or } \\
\text { equal to threshold value } \tau_{r e f}\end{array}$ \\
\hline
\end{tabular}

\begin{tabular}{|l|l|}
\hline$\Gamma$ & $\begin{array}{l}\text { Set of all clusters (the number of clusters and the number of a } \\
\text { given cluster's elements are not specified a priori) } \\
\tau_{k}\end{array}$ \\
$\begin{array}{l}\text { Radius of hypersphere or immediate neighborhood zone to be } \\
\text { scanned (centered around a point).i.e. } \tau_{k}=2\end{array}$
\end{tabular}

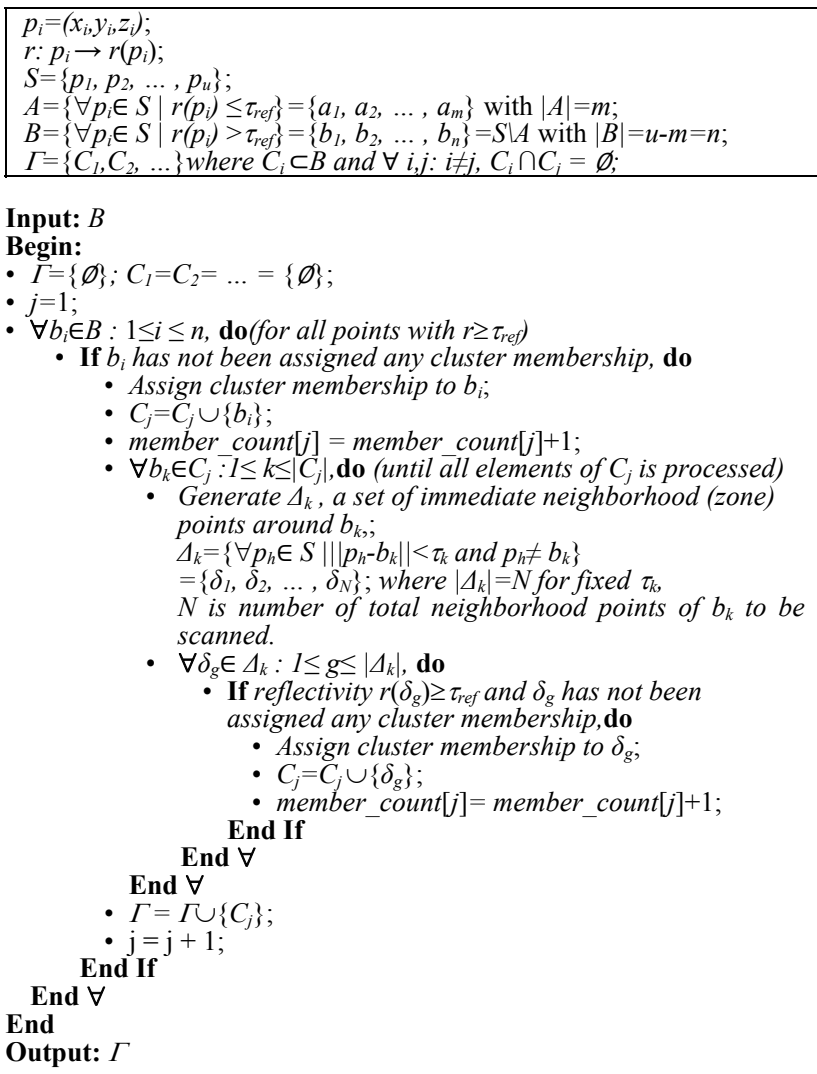

\subsection{Simulation Results}

Table 1. Simulation Environment

\begin{tabular}{|c|c|}
\hline $\begin{array}{c}\text { Environment } \\
\text { Settings }\end{array}$ & Description \\
\hline Radar Polarization & Horizontal \\
\hline Test Radar Site & KSN \\
\hline CPU & Intel(R) Core(TM)i5 M4802.67GHz \\
\hline RAM & $3072 \mathrm{MB}$ \\
\hline Disk Space & $80 \mathrm{~GB}$ \\
\hline OS & Linux Ubuntu 10.10 \\
\hline
\end{tabular}

Table 2. Simulation Parameters

Table 2. Simulation Parameters
\begin{tabular}{|c|c|}
\hline Parameters & Value \\
\hline $\begin{array}{c}\text { Input CZ Radar Volume Size }(1 \\
\text { byte per radar data point) }\end{array}$ & $\mathrm{R} \times 0 \times \phi: 980 \times 360 \times 15$ \\
Total: $5,292,000$ bytes \\
\hline $\begin{array}{c}\text { Input 3D Grid Space Size }(1 \text { byte } \\
\text { per grid or data point) }\end{array}$ & $\mathrm{X} \times \mathrm{Y} \times \mathrm{Z}: 480 \times 480 \times 41$ \\
Total: $9,446,400$ bytes \\
\hline 3D Input Grid Resolution & $\mathrm{X} \times \mathrm{Y} \times \mathrm{Z}: 1 \mathrm{~km} \times 1 \mathrm{~km} \times 0.25 \mathrm{~km}$ \\
\hline Sampling Date & 2010.05 .19 \\
\hline & From 10:00 To $16: 30$ \\
Sampling Time & At 10 min interval \\
& Total of 40 time frames \\
\hline$Z_{t h}$ (Threshold CZ reflectivity) & 0 \\
\hline$S_{t h}$ (Threshold cluster size) & 189 data points \\
\hline$\tau_{k}$ (Radius of hyper-sphere) & 2 grid distance \\
\hline
\end{tabular}


To evaluate the performance of the zone-linkage clustering method, a simulated experiment is carried out with simulation environment settings and parameters shown in Table 1 and Table 2.

Performance comparison between single-pass and zonelinkage clustering methods is conducted by running each algorithm over 40 time frame sequences and averaging out the execution time - total clustering time taken divided by the number of time frames sequences- while retaining the same parameter settings. The result is shown in Table 3.

Table 3. Simulation Results

\begin{tabular}{|c|c|c|}
\hline Time Measurements & Single-Pass & Zone-Linkage \\
\hline $\begin{array}{c}\text { Total Clustering Time } \\
\text { (for 40 time frames) }\end{array}$ & $\begin{array}{c}41 \mathrm{~min} 21.73 \\
\mathrm{sec}\end{array}$ & $19.40 \mathrm{sec}$ \\
\hline $\begin{array}{c}\text { Average Clustering Time (per } \\
\text { time frame) }\end{array}$ & $1 \mathrm{~min} 2.04 \mathrm{sec}$ & $0.485 \mathrm{sec}$ \\
\hline $\begin{array}{c}\text { Total Time Taken by } \\
\text { Coordinate Transformation } \\
\text { (for 40 time frames) }\end{array}$ & $3 \mathrm{~min} 7.11 \mathrm{sec}$ \\
\hline $\begin{array}{c}\text { Average Time Taken by } \\
\text { Coordinate Transformation } \\
\text { (per time frame) }\end{array}$ & $4.68 \mathrm{sec}$ \\
\hline
\end{tabular}

Overall data partition or decomposition of 3D radar image that results from reflectivity range and size range filtering is illustrated in Fig. 2.
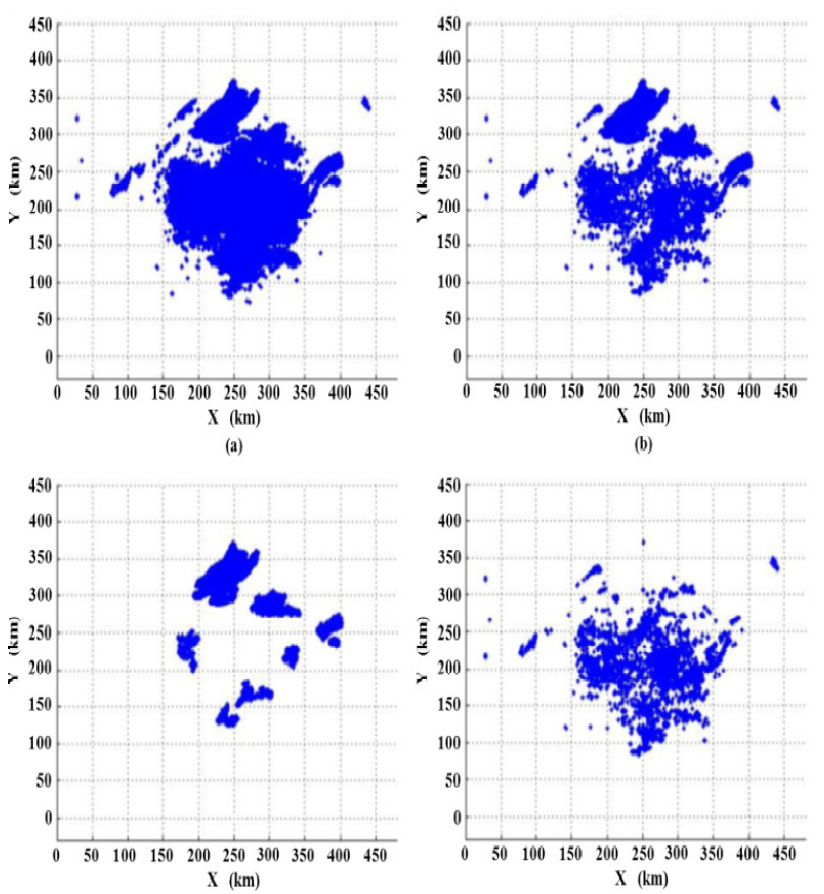

(d)

Fig.2. 3D radar image decomposition (KSN 2010-05-19 15:00).

(a) full echoes. (b) below-threshold-reflectivity echoes removed. (c) below-threshold-reflectivity echoes, below-threshold-size clusters removed. Total of 8 clusters remained.

(d) below-threshold-reflectivity echoes, above-threshold-size clusters removed.

\section{Dynamic Frame Clustering Method}

\subsection{Description of Evolution-Linkage Clustering Method}

The evolution-linkage clustering procedure is performed with only reasonably sized clusters from previous step and intrinsically associates similar clusters in subsequent time frames into one group forming a subspace called iso-cluster set. The evolution-linkage uses the distance that separates clusters' centroids and clusters' size ratio(either volume ratio or planar projection area ratio) as the basis for similarity comparison. Other arbitrary features such as reflectivity profile can be used as comparison criteria. However, for the purpose of simultaneous monitoring of many cells at optimum frame rates, these features may run redundant. Cluster splitting is weighed in the algorithm as the algorithm starts new linkage chain of cells at the split point and tracks it as a separate disjoint isocluster set with its own evolving characteristic features. Cluster merging is handled by joining two separate iso-cluster sets into one and tracking it as a combined feature set. The algorithm assigns iso-cluster membership to every clusters within inference time frames and no two clusters within single time frame will have the same iso-cluster membership.

\subsection{Evolution-Linkage Clustering Algorithm}

Given:

\begin{tabular}{|l|l|}
\hline$t, v$ & Time frame index \\
$\kappa, l$ & Cluster id \\
$\mu$ & 2-tuple cluster identifier \\
$T$ & Number of inference time frames \\
$N$ & Total cluster count in all inference time frames \\
$L(t)$ & Total cluster count in single time frame $t$ \\
$\rho$ & Centroid of cluster with $\mu$ identifier \\
$\sigma$ & Size of cluster with $\mu$ identifier \\
$\Pi$ & Set of all cluster identifiers within inference time frames $T$ \\
$\Phi$ & Set of all clusters with below-or-equal-to-threshold-size \\
$\Psi$ & Set of all clusters with above-threshold-size \\
$\Omega$ & Set of all iso-clusters \\
$w$ & iso-cluster set (set of 2-tuple cluster identifiers) \\
$\lambda_{t}$ & Set of all cluster id for above-threshold-size category at time \\
& frame $t$ \\
$\Xi$ & Set of all $\lambda_{t}$ within inference time frames \\
$d_{k}$ & Distance between centroids \\
$h_{k}$ & Ratio of cluster size valued between 0 and 1 \\
$G_{k}$ & Final Grade \\
$Q$ & Similarity Function \\
\hline
\end{tabular}

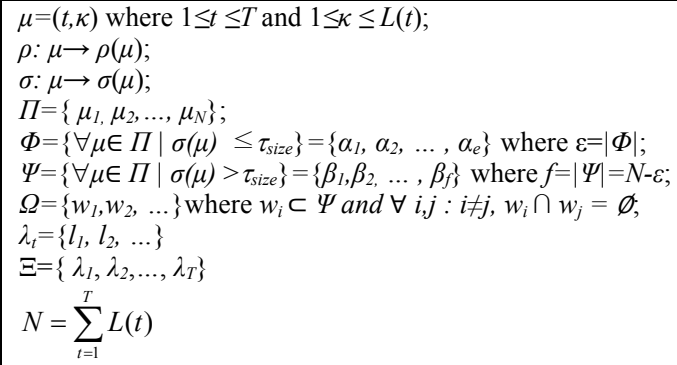




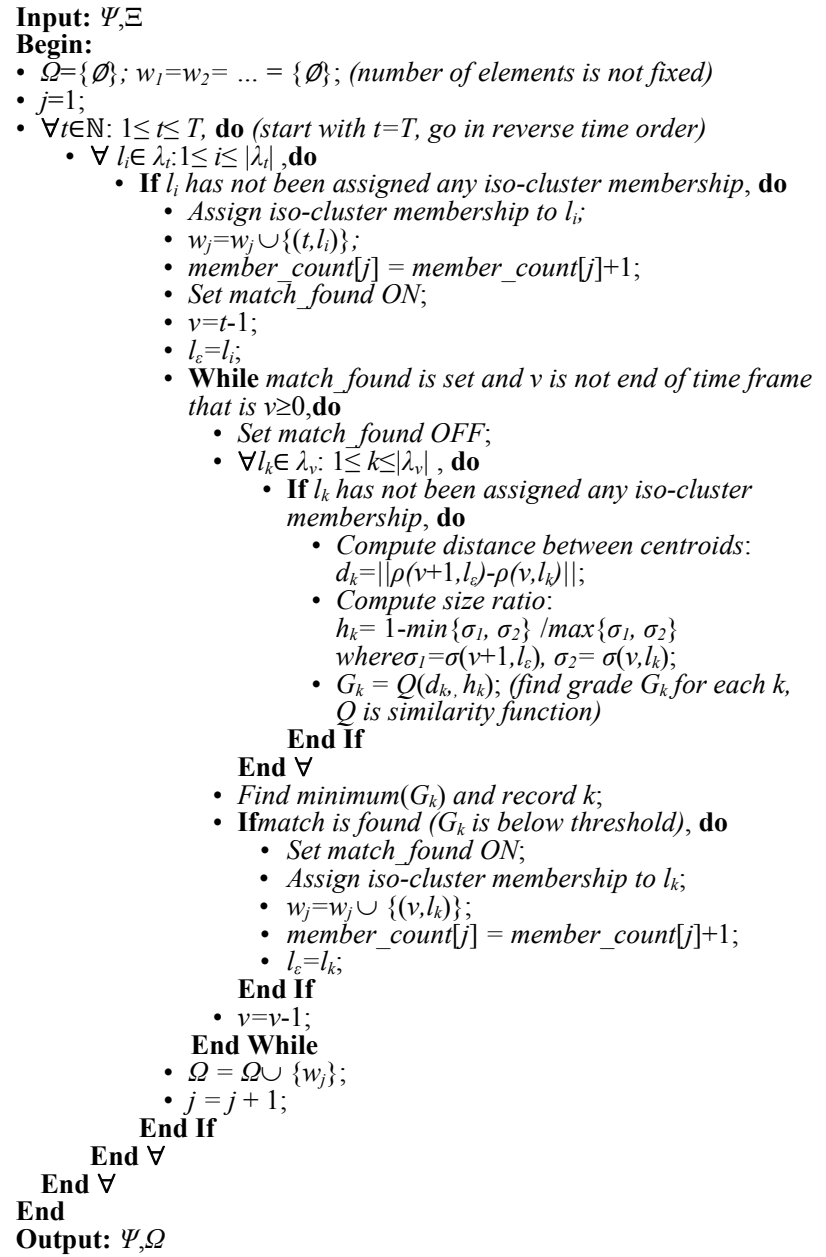

\subsection{Similarity Measure}

Given two clusters $\mathrm{C}_{1}, \mathrm{C}_{2}$ in two adjacent time frames and their respective centroids $\mathrm{p}_{1}, \mathrm{p}_{2}$ the distance that separates these two centroids is given by $d=\left\|p_{1}-p_{2}\right\|$. The size ratio between two clusters is determined by $\mathrm{r}_{\mathrm{s}}=1-\min \left\{\mathrm{v}_{1}, \mathrm{v}_{2}\right\} / \max \left\{\mathrm{v}_{1}, \mathrm{v}_{2}\right\}$ where $\mathrm{v}_{1}, \mathrm{v}_{2}$ is cluster's respective volume (or planar projection area). These two variables are then rendered fuzzy by using membership functions shown in Fig. 3.
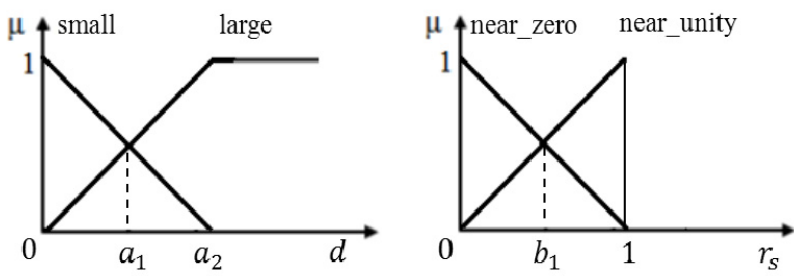

Fig.3. Two input fuzzy variables $d$ and $r_{s}$ in the antecedent with their MFs.

Based on these two fuzzy variables, the following two-input single-output TSK fuzzy model with four rules is constructed. In this model, the output $(\mathrm{G} \in[0,1])$ represents the degree of similarity between two clusters. The higher the degree (unity being the maximum), the less similar two clusters are.
If $\mathrm{d}$ is small and $\mathrm{r}_{\mathrm{s}}$ is near_zero Then $G=\left\{\begin{array}{cc}\frac{d}{2 a_{2}}+\frac{r_{s}}{2} & 0<d<a_{2} \\ 1 & d>a_{2}\end{array}\right.$

If $\mathrm{d}$ is small and $\mathrm{r}_{\mathrm{s}}$ is near_unity Then $G=\left\{\begin{array}{cc}\frac{d}{2 a_{2}}+\frac{1}{2} & 0<d<a_{2} \\ 1 & d>a_{2}\end{array}\right.$

If $\mathrm{d}$ is large and $\mathrm{r}_{\mathrm{s}}$ is near_zero Then $G=\frac{1}{2}+\frac{r_{s}}{2}$

If $\mathrm{d}$ is large and $\mathrm{r}_{\mathrm{s}}$ is near_unity Then $G=1$

Since each rule has a crisp output, the overall output is obtained via the weighted average. This enables sample data based fuzzy modeling without the time-consuming and mathematically intractable defuzzification operation. The above TSK fuzzy system can be represented as ANFIS shown in Fig. 4.

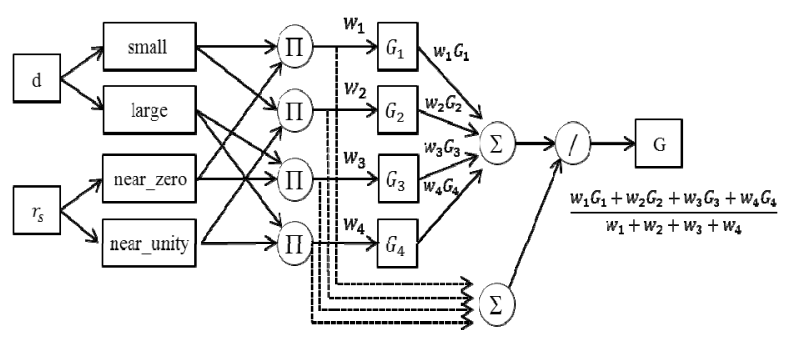

Fig.4. ANFIS for the determination of the degree of similarity

\subsection{Simulation Results}

The performance test of the evolution-linkage clustering is carried out with same simulation environment settings and parameters as in Table 1 and Table 2 over 6 hours of inference time period. KSN radar site data dating back to 2010.05.19 is used for test simulation. The result is shown in Table 4.

Table 4. Simulation Result of Evolution-Linkage Clustering

\begin{tabular}{|c|c|}
\hline Parameters & Value \\
\hline Inference Time & 360 min (6 hours) \\
\hline Total Inference Time Frames & 37 \\
\hline Sampling Time & $\begin{array}{c}\text { From 15:00 To 16:30 } \\
\text { At 10 min interval }\end{array}$ \\
\hline Total Sampling Time Frames & 10 \\
\hline $\begin{array}{c}\text { Total Clustering Time } \\
\text { for total sampling time frames) }\end{array}$ & $5.54 \mathrm{sec}$ \\
\hline $\begin{array}{c}\text { Average Clustering Time (per } \\
\text { sampling time frame) }\end{array}$ & $0.554 \mathrm{sec}$ \\
\hline
\end{tabular}

\section{Reflectivity Profile}

The characterization of the convective activity of cloud cells is an important subject in meteorology. Cold convective clouds often convey severe weather situations in the form of pouring rains or even microbursts. Reflectivity profile of rain echoes 
provides means to assess rapidly changing weather situation by enabling one to estimate the amount of precipitation in selected regions. Time series profile of reflectivity distribution can be used to determine the expansion rate of given convective cloud cells as well as any potential changes in precipitation conditions.

Fig. 5 shows such profile for anomalous weather echoes (single cluster) found in the midst of other residual background echoes on May 19, 2010 by KSN radar.
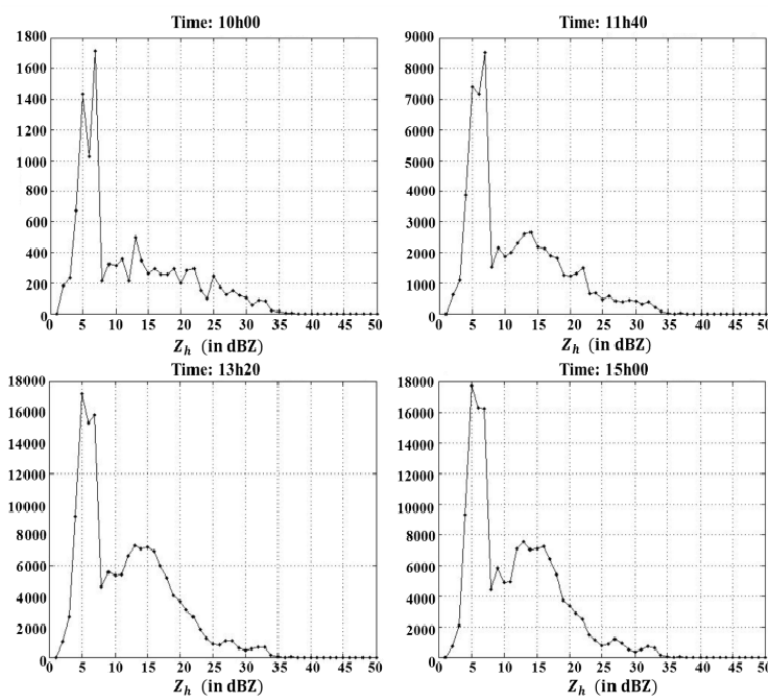

Fig.5. Time series profile of reflectivity distribution of anomalous weather echoes

\section{Conclusion}

In this paper, a novel technique for tracking and characterizing deformable 3D radar structures is proposed. The characterization of the tracked cloud cells relies on both 3D localization of cloud centers, overall volume expansion rate and temporal variations of its reflectivity or rain density profile. The statistical labeling of changing weather events based on contextual awareness can be applied to six categories of events: "severe activity", "emerging activity", "growing activity", "declining activity", "inactivity" and "anomalous activity". The two clustering schemes proposed here are applied on real radar data (in UF format) provided by KMA. Multi-sites numerical simulations show the validity of the proposed technique and that fast and accurate characterization of deformable 3D radar structures is achievable through time series profiling of reflectivity distribution.

\section{References}

[1] W.I. Rose, A.B. Kostinski, and L. Kelley, "Real-Time CBand Radar Observations of 1992 Eruption Clouds from Crater Peak, Mount Spurr Volcano, Alaska," US Geol. Surv.
Bull., vol. 2139, pp. 19-26, 1995.

[2] V.N. Bringi and V. Chandrasekar, "Polarimetric Doppler Weather Radar: Principles and Applications," Cambridge, UK: University Press, pp. 648, 2001.

[3] S.T. Barnard and W.B. Thompson, "Disparity analysis of images," IEEE Trans. Pattern Anal. Machine Intell., vol. 2, pp. 333-340, 1980.

[4] H. Zhang, "Storm detection in radar images," Master's Thesis, Department of Computer Science, University of Western Ontario, 1991.

[5] D. Krezeski, R.E. Mercer, J.L. Barron, P. Joe, and H. Zhang, "Storm tracking in Doppler radar images," Proc. of the International Conference on Image Processing (ICIP94), vol. 3, pp. 226-230, 1994.

[6]R.C. Tryon, Cluster Analysis, Ann Arbor, MI:Edward Brothers, 1939.

[7] J.A. Hartigan, "Clustering Algorithms," Wiley, New York, 1975.

[8] F. Murtagh, "Multidimensional clustering algorithms," CompStat Lectures, vol. 4, Physica Verlag, Mostbach, 1985.

[9] I. Karkkainen and P. Franti, "Gradual model generator for single pass clustering," The Journal of The Pattern Recognition Society, vol. 4, pp. 784-795, 2007.

[10] S.H. Wei and S.M. Chen, "A New Similarity Measures Between Interval-valued Trapezoidal Fuzzy Numbers Based on Geometric Distance and the Center-of-gravitypoints," Proc. of the2007 Sixth Int'l Conf. on Machine Learning and Cybernetics, Hong Kong, China, pp.1412$1417,2007$.

[11] H. Bae, S. Kim, G. Vachtsevanos,"Fault Detection and Diagnosis of Winding Short in BLDC Motors Based on Fuzzy Similarity," Int. Journal of Fuzzy Logic and Intelligent Systems, vol.9, no.2, pp.99-104, 2009.

[12] G. J. Ryu, S. G. Park, J. P. Hwang, E.T. Kim, H. J. Gang, "Grouping Radar Sensor Data for Detecting Object," Proc. of the Korean Institute of Intelligent Systems Conference, pp. 394-396, 2007.

[13] D. W. Kim. "On Color Cluster Analysis with Threedimensional Fuzzy Color Ball," Journal of Korean institute of intelligent Systems, vol.18, no.2, pp.262-267, 2008

[14] D. W. Kim, K. H. Lee."A Cluster Validity Index Using Overlap and Separation Measures between Fuzzy Clusters," Journal of Korean institute of intelligent Systems, vol.13, no.4, pp.455-460, 2003

[15] D. W. Kim, K. H. Lee. "A Fuzzy Cluster Validity based on Inter-cluster Overlapping and Separation," Journal of Korean institute of intelligent Systems, pp.99-102, 2003

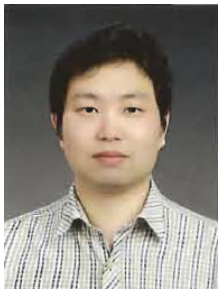

Yong Hyun Kim

He received the B.S. degree in Physics from McGill University, Montreal, Canada, in 2001. He is currently pursuing the M.S. degree at Pusan National University. His previous employers include McGill Dep. of Anesthesia Research and Canadian Space 
Agency. His research interests include intelligent control, pattern recognition, robotics and remote sensing.

Phone: +82-10-9264-7391

Fax:+82-51-513-0212

E-mail: yhkim@pusan.ac.kr

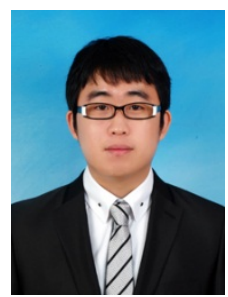

Hansoo Lee

He received the B.S. degree in Electrical Engineering from Pusan National University, Busan, Korea, in 2010. He is currently pursuing the M.S. degree at Pusan National University. His research interests include intelligent control, pattern recognition, data mining and remote sensing.

Phone: +82-10-6788-9372

Fax:+82-51-513-0212

E-mail: hansoo@pusan.ac.kr

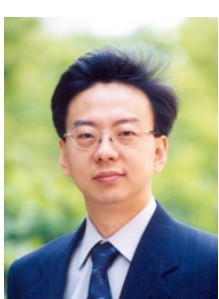

\section{Sungshin Kim}

He received the B.S. and M.S. degrees in electrical engineering from Yonsei University, Seoul, Korea, in 1984 and 1986, respectively, and the Ph.D. degree in electrical and computer engineering from Georgia Institute of Technology, Atlanta, in 1996. He is currently Professor in the School of Electrical Engineering, Pusan National University, Busan, Korea. His research interests include intelligent control, fuzzy logic control, hierarchical learning structures and data mining. Phone: +82-51-510-2374

Fax:+82-51-513-0212

E-mail: sskim@pusan.ac.kr 